\title{
Stroboscopic Evaluation of the Vocal Folds' Morphometric Characteristics, between the Different Voice Categories on Professional Singers
}

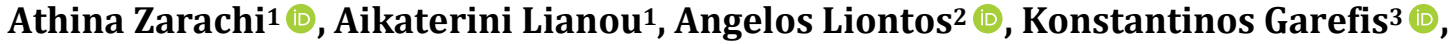 \\ Eleni Litsou1, Dionysios Tafiadis, ${ }^{4,5}$, Efthymis Dimakis6, Ioannis Kastanioudakis', \\ Georgios Exarchakos ${ }^{1}$
}

\begin{abstract}
${ }^{1}$ Department of Otorhinolaryngology, Head and Neck Surgery, Faculty of Medicine, School of Health Sciences, University of Ioannina, Ioannina, Greece

${ }^{2}$ Department of Internal Medicine, Faculty of Medicine, School of Health Sciences, University of Ioannina, Ioannina, Greece

${ }^{3}$ 2nd Academic ORL, Head and Neck Surgery Department, Papageorgiou Hospital, Aristotle University of Thessaloniki,

Thessaloniki, Greece

${ }^{4}$ Department of Speech \& Language Therapy, School of Health Sciences, University of Ioannina, Ioannina, Greece

${ }^{5}$ Department of Health Sciences, Speech and Language Therapy, European University Cyprus, Nicosia, Cyprus

${ }^{6}$ Division of Biostatistics and Epidemiology, Department of Medical School, University of Ioannina, Ioannina, Greece

Email: athinazarachi@gmail.com, lianoynikoletta@yahoo.gr, elenilitsou@gmail.com, ikastan@uoi.gr,

exarchakos.george@gmail.com, angelosliontos@gmail.com, kgarefis@hotmail.com, tafiadis@uoi.gr,

efdimakis@gmail.com
\end{abstract}

How to cite this paper: Zarachi, A., Lianou, A., Liontos, A., Garefis, K., Litsou, E., Tafiadis, D., Dimakis, E., Kastanioudakis, I. and Exarchakos, G. (2021) Stroboscopic Evaluation of the Vocal Folds' Morphometric Characteristics, between the Different Voice Categories on Professional Singers. International Journal of Otolaryngology and Head \& Neck Surgery, 10, 345-358. https://doi.org/10.4236/ijohns.2021.105031

Received: June 24, 2021

Accepted: August 6, 2021

Published: August 9, 2021

Copyright $\odot 2021$ by author(s) and Scientific Research Publishing Inc. This work is licensed under the Creative Commons Attribution International License (CC BY 4.0).

http://creativecommons.org/licenses/by/4.0/

\begin{abstract}
Objective(s): The aim of this study is to explore if there is a correlation between the typical voice classification and the morphometric characteristics of the vocal folds, using video laryngeal stroboscopy, on professional singers in Greece. Methods: 70 professional singers, 50\% men (35 men) and 50\% women (35 women), were recruited for this study. A stroboscopic evaluation was prepared for every participant. Additionally, the voice classification and features of individuals were correlated statistically. Results: Statistically significant difference were observed between the voice categories and the size of stomatopharynx, the shape of the hard palate, the length of the vocal folds, the width and thickness of the vocal folds, as well as the tone of speech, in male singers. Statistically significant differences were also observed between the voice categories and all the variables except the shape of epiglottis, the color of speech and the shape of the vocal folds, in female singers. Conclusions: There is correlation between the morphometric characteristics of the vocal folds and the voice categories.
\end{abstract}




\section{Keywords}

Stroboscopy, Vocal Folds, Professional Singers, Voice Category, Morphometric Characteristics

\section{Introduction}

Considering voice as the generator of voiced sound, it can be functionally separated into three units: the lungs that provide the power supplies, the vocal folds that use as oscillators and the vocal tract, which works as a resonator [1]. The singing voice production depends on many parameters, such as developmental, physical, psychological, environmental and the vocal training [2] [3] [4] [5] [6]. Singing voice has prevailed to be classified into groups, based on the frequency range of each singer, as follows: male voices belong to one of the categories basso, tenor, baritone, while female voices belong to one of the categories contralto, mezzo-soprano, and soprano [7] [8] [9] [10]. Many researchers have attempted to quantify the factors that influence and form phonation and singing, providing special methods of examination. So, radiological methods, such as the x-ray imaging, the computed tomography, the magnetic tomography, as well as the ultrasound-imaging test have been used as methods for the examination of larynx, over the years [11]-[16]. Most of the studies correlate the classical categories with body type, improving that low-frequency voices (such as bass male singers) tend to correlate with taller people, while high-frequency voices (such as tenor male singers and soprano female singers) correlate with shorter people [17]. Furthermore, other studies have suggested that there is a relationship between laryngeal size (length, shape and thickness of the vocal folds) and qualitative vocal characteristics (pitch, loudness and timbre), as they are observed clinically by vocal coaches and ENT doctors. The morphological characteristics of the vocal folds seem to differ between voice categories, and affect the voice production while singing [18] [19] [20] [21]. Our purpose was to evaluate the vocal folds' characteristics, using the examination method of stroboscopy.

\section{Materials and Methods}

\subsection{Participants}

Seventy (70) healthy singers (male: 35; female: 35), aged from 18 to 70, were included in this study. All of the participants were professional singers that practice voice in specialized centers, in Ioannina, Greece. Especially, all of the participants belong to the choirs of the municipality or practice in any of the conservatories in Ioannina city. The thirty-five males were classified as follows: 1) 16 basses, 2) 11 baritones, and 3) 8 tenors. Likewise, the thirty-five females were classified as: 1) 7 contraltos, 2) 12 mezzo-sopranos and 3) 16 sopranos. All subjects were classified into singing groups by an experienced vocal coach. Specific 
included and excluded criteria were used to determine the final sample. All singers with a medical history of head and neck surgery or neck radiation were not included in this study [22] [23] [24] [25]. Additionally, subjects who had presented during the previous weeks laryngeal/vocal complaints, symptoms of gastro re-flux (GERD), or laryngopharyngeal reflux (LRP) disease, were excluded from this study [26] [27] [28] [29] [30]. Finally, subjects with a medical history of voice disorders in the past, of abuses (e.g. alcohol, drugs), smokers, were also excluded from the study [31]-[37]. Finally, female participants that were pregnant were also excluded from the study, because their receiving any radioactive substance would have been impermissible [38] [39]. This study was approved by the Ethical Committee of the Medical School, University of Ioannina.

\subsection{Collection of Data}

The above data were obtained through a specific history form used in the ENT clinic of the University Hospital of Ioannina. The sample was evaluated at the ENT clinic of the University Hospital of Ioannina, Greece. Each subject was informed of the research purposes and gave a written consent. We used a video laryngeal stroboscopy for each participant, for the evaluation of the vocal folds.

\subsection{Video Laryngeal Stroboscopy}

Each professional singer was submitted to a stroboscopic evaluation of the larynx. The choice of this endoscopic method was held because of some specific advantages. It is a non-invasive method of introspection of vocal folds that provides the opportunity of detailed imaging of the vocal folds' mobility and movement of the mucosal wave [40]-[45]. During stroboscopy, each singer was asked to produce an /e/ sound at a frequency of tessitura. Tessitura refers to the the most comfortable modal singing voice range for the professional singer [46] [47] [48]. Through the laryngeal stroboscopy, we selected specific information about: the shape of the vocal folds (round shaped, striped shaped), the shape of the epiglottis (flat, omega shaped), the length of the vocal folds (short, medium, long), the width of the vocal folds (narrow, wide, medium), the thickness of the vocal folds(thin, coarse, medium). We used a rigid transoral Storz Stroboscope for the examination of subjects.

\subsection{Nasal-Sinus and Clinical Evaluation}

A clinical evaluation of all participants was also performed from an ENT doctor to record the size of the oral cavity (small, medium, large) and the shape of the hard palate (oval, arched, flat). Through a subjective clinical examination we also observed the color of the voice (light, dark) and the tone of speech (acute, medium, heavy), of every participant, as qualitative data. An anterior rhinoscopy, using a small-sized Killian's Nasal Speculum and a headlight, was finally used to evaluate the transparency of the nasal cavity (good, reduced) [49]. 


\subsection{Statistical Analysis}

The distribution of variables was tested using the Kolmogorov-Smirnov and Shapiro-Wilk tests. All skewed variables are expressed as the medians and the inter-quartile range, and all normally distributed variables are expressed as the means and standard deviations (SD). Spearman's rank correlation coefficient was used to correlate the vocal category of the participants and the permeability of nasal cavity, oral pharyngeal size, shape of the hard palate, epiglottis, length of the vocal chords, shape of the vocal chords, width of the vocal chords, thickness of the vocal chords, tone/tonicity of the speech, vocal timbre. All reported pvalues were two-tailed, and the statistical significance was set at the value of $\mathrm{p}<$ 0.05. The analysis was performed with SPSS statistical software (version 19.0, Armonk, NY, USA).

\section{Results}

\section{Subsection}

Through the examination of gender percentages, the participants are divided into: 50\% men ( 35 men) and 50\% women ( 35 women). Regarding the voice category to which the participants belong, the analysis showed that 16 singers (22.9\%) belong in the bass category, 11 singers (15.7\%) are baritones and $8(11.4 \%)$ are tenors. 7 singers $(10 \%)$ are contralto, $12(17.1 \%)$ are mezzo-soprano and 16 singers (22.9) are soprano. The mean age of the total sample was 38.64 years (SD $=16.52)$ with the mean age of males at $36.51(\mathrm{SD}=14.34)$ and the mean age of females at 40.77 years $(S D=18.41)$. The mean height of the total sample was 1.73 meters $(\mathrm{SD}=0.08)$ with that for males being $1.76(\mathrm{SD}=0.06)$ and for females 1.70 ( $\mathrm{SD}=0.08)$. The mean body weight of the total sample was $72.46 \mathrm{~kg}$ $(\mathrm{SD}=10.88)$ with male mean body weight of $76.71(\mathrm{SD}=9.96)$ and female mean body weight $68.20 \mathrm{~kg}(\mathrm{SD}=10.19)$. The mean years of vocal training for the total sample was 9.26 years $(\mathrm{SD}=7.13)$ with mean male vocal training at $9.63(\mathrm{SD}=$ 7.74) and female mean vocal training at 8.89 years $(\mathrm{SD}=6.95)$.

In an additional analysis, we correlated the weight and the age with the voice category. A non-statistically significant difference was observed for the age $[\mathrm{p}=$ 0.465]. A statistically significant difference was observed for the height $[\mathrm{F}=$ $35.22, \mathrm{p}=0.000]$ and the difference was between the bass and baritone $[\mathrm{p}=$ $0.000]$ and between the bass and the tenor $[p=0.000]$. Statistically significant differences were also observed between the weight $[\mathrm{F}=28.76, \mathrm{p}=0.000]$ and the difference was between bass and baritone $[p=0.001]$, bass and tenor $[p=0.000]$ and between baritone and tenor $[\mathrm{p}=0.009]$. Regarding the female voice categories, the same statistical analysis was followed. A non-statistically significant difference was observed for the age $[p=0.826]$. A statistically significant difference was observed for the average height in centimeters $[p=0.000]$ with the difference being between contralto and mezzo-soprano $[p=0.000]$ and between mezzo-soprano and soprano [ $p=0.048]$. Statistically significant differences were also observed between the average weight $[p=0.000]$ with the difference being be- 
tween loud and acute loud $[\mathrm{p}=0.000]$.

The objective examination showed that 9 basso, 5 baritones and 6 tenors of the group of male singers had good transparency of the nasal cavity, while 7 basso, 6 baritones and 2 tenors had reduced transparency. All of 16 basso singers appeared large stomatopharynx. 1 baritone appeared small stomatopharynx, 9 baritones appeared mean size, while 1 appeared large stomatopharynx. All of 8 tenors appeared small stomatopharynx (Table 1 ).

The shape of hard palate was evaluated next. 13 basso singers appeared an oval-shaped palate, 3 basso presented arched palate and no one appeared flat hard palate. 5 baritones appeared an oval-shaped palate, 4 baritones presented arched palate and 2 appeared flat hard palate. 2 tenors appeared an oval-shaped palate, 1 presented arched palate and 5 appeared flat hard palate. Regarding the shape of the epiglottis, 7 basso, 6 tenors and had a high-rising epiglottis, while 5 basso, 5 baritones and 2 tenors had a lower-lying epiglottis (Table 2).

Concerning the length of vocal folds, in male singers, all of tenors appeared short vocal folds, while all basso appeared long vocal folds. Finally, 9 baritones appeared meddle length of vocal folds, while 2 baritones appeared long vocal folds. In terms of the shape of the vocal folds in male singers, 9 basso appeared rounded shape and 7 basso appeared elongated. 5 baritones appeared rounded and 6 appeared elongated vocal folds. Finally, 5 tenors appeared rounded and 3 appeared elongated vocal folds (Table 3).

Concerning the width of the vocal folds of the male singers, all of 15 basso appeared wide vocal fold. 2 baritones appeared wide vocal folds, 9 baritones appeared medium size vocal folds and 8 baritones appeared narrow vocal folds. The thickness of the vocal folds was found to be thin in 2 basso singers and 8 tenors. 14 basso, 2 baritones appeared thick vocal folds and 9 baritones appeared average thickness (Table 4).

The tone of speech was heavy in all of the 16 basso and in 2 baritones. 9 baritones appeared average tone of speech. All of the 8 tenors appeared acute tone of

Table 1. Statistical analysis results: correlation between male singers and the variables; reliability of the nasal cavity, size of stomatopharynx.

\begin{tabular}{cccccccc}
\hline voice category & \multicolumn{2}{c}{ reliability of nasal cavity } & \multicolumn{3}{c}{ size of stomatopharynx } \\
\hline & good & reduced & total & small & Mean & large & total \\
\hline basso & 9 & 7 & 16 & 0 & 0 & 16 & 16 \\
baritones & $56.30 \%$ & $43.80 \%$ & $100 \%$ & $0 \%$ & $0.00 \%$ & $100 \%$ & $100 \%$ \\
& 5 & 6 & 11 & 1 & 9 & 1 & 11 \\
tenors & $45.50 \%$ & $54.50 \%$ & $100 \%$ & $9.10 \%$ & $81.80 \%$ & $9.10 \%$ & $100 \%$ \\
& 6 & 2 & 8 & 8 & 0 & 0 & 8 \\
Pearson Chi-square & $75 \%$ & $25 \%$ & $100 \%$ & $100 \%$ & $0 \%$ & $0 \%$ & $100 \%$ \\
p-level & & 1.661 & & & 58.22 & & \\
\hline
\end{tabular}


Table 2. Statistical analysis results: correlation between male singers and the variables; shape of hard palate, shape of epiglottis.

\begin{tabular}{cccccccc}
\hline voice category & \multicolumn{3}{c}{ shape of hard palate } & \multicolumn{3}{c}{ shape of epiglottis } \\
\hline & oval & arched & flat & total & high & lower & total \\
\hline basso & 13 & 3 & 0 & 16 & 7 & 9 & 16 \\
baritones & $81.30 \%$ & $18.80 \%$ & $0 \%$ & $100 \%$ & $48 \%$ & $56.30 \%$ & $100 \%$ \\
& 5 & 4 & 2 & 11 & 6 & 5 & 11 \\
tenors & $45.50 \%$ & $36.40 \%$ & $18.20 \%$ & $100.00 \%$ & $55 \%$ & $45.50 \%$ & $100 \%$ \\
Pearson Chi-square & 2 & 1 & 5 & 8 & 6 & 2 & 8 \\
p-level & $25 \%$ & $13 \%$ & $63 \%$ & $100 \%$ & $70 \%$ & $25 \%$ & $100 \%$ \\
\hline
\end{tabular}

Table 3. Statistical analysis results: correlation between male singers and the variables; length of vocal folds, shape of vocal folds.

\begin{tabular}{|c|c|c|c|c|c|c|c|}
\hline \multirow[t]{2}{*}{ voice category } & \multicolumn{4}{|c|}{ length of vocal folds } & \multicolumn{3}{|c|}{ shape of vocal folds } \\
\hline & short & meddle & long & total & Rounded & elongated & total \\
\hline \multirow[t]{2}{*}{ basso } & 0 & 0 & 16 & 16 & 9 & 7 & 16 \\
\hline & $0 \%$ & $0 \%$ & $100 \%$ & $100 \%$ & $56.30 \%$ & $43.80 \%$ & $100 \%$ \\
\hline \multirow[t]{2}{*}{ baritones } & 0 & 9 & 2 & 11 & 5 & 6 & 11 \\
\hline & $0 \%$ & $81.80 \%$ & $18.20 \%$ & $100 \%$ & $45.50 \%$ & $54.50 \%$ & $100 \%$ \\
\hline \multirow[t]{2}{*}{ tenors } & 8 & 0 & 0 & 8 & 5 & 3 & 8 \\
\hline & $100 \%$ & $0 \%$ & $0 \%$ & $100 \%$ & $62.50 \%$ & $37.50 \%$ & $100 \%$ \\
\hline Pearson Chi-square & & 60.45 & & & & 0.58 & \\
\hline p-level & & 0 & & & & 0.745 & \\
\hline
\end{tabular}

Table 4. Statistical analysis results: correlation between male singers and the variables; width of vocal folds, thickness of vocal folds.

\begin{tabular}{cccccccccc}
\hline voice category & \multicolumn{3}{c}{ width of vocal folds } & \multicolumn{5}{c}{ thickness of vocal folds } \\
\hline & wide & meddle & narrow & total & thin & average & thick & total \\
\hline basso & 15 & 0 & 1 & 16 & 2 & 0 & 14 & 16 \\
baritones & $93.80 \%$ & $0 \%$ & $6.30 \%$ & $100 \%$ & $12.50 \%$ & $0 \%$ & $87.50 \%$ & $100 \%$ \\
tenors & 2 & 9 & 0 & 11 & 0 & 9 & 2 & 11 \\
& $18.20 \%$ & $81.80 \%$ & $0 \%$ & $100 \%$ & $0 \%$ & $81.80 \%$ & $18.20 \%$ & $100 \%$ \\
Pearson Chi-square & 0 & 0 & 8 & 8 & 8 & 0 & 0 & 8 \\
p-level & $0 \%$ & $0 \%$ & $100 \%$ & $100 \%$ & $100 \%$ & $0 \%$ & $0 \%$ & $100 \%$ \\
\hline
\end{tabular}

speech. Finally, in observing the color of each participant's speech, it was found that 11 basso, 5 baritones and 4 tenors exhibited deep voice color. 5 basso, 6 ba- 
ritones and 4 tenors appeared high-tone voice color (Table 5).

The objective examination based on stroboscopy clinical findings in female singers, showed that: 2 contralto, 4 mezzo-soprano and 14 soprano had good transparency of the nasal cavity, while 5 contralto, 8 mezzo-soprano and 3 soprano had reduced transparency. All of 7 contralto singers appeared large stomatopharynx, all of 12 mezzo-soprano singers appeared mean size of stomatopharynx, while all of the 16 soprano singers appeared large stomatopharynx (Table 6).

The shape of hard palate in female singers was evaluated next. 5 contralto appeared an oval-shaped palate and 2 contralto had flat hard palate. 3 mezzo-soprano appeared oval-shaped palate, 7 mezzo-soprano appeared arched palate and 2 appeared flat hard palate. 2 soprano appeared an oval-shaped palate, 5 presented arched palate and 9 soprano appeared flat hard palate. Regarding the shape of the epiglottis, 1 contralto, 8 mezzo-soprano and 10 soprano had a high-rising epiglottis. 5 basso, 56 contralto, 4 mezzo-soprano and 4 soprano had a lower-lying epiglottis (Table 7).

Concerning the length of vocal folds, in female singers, all of contralto appeared long vocal folds. All of the mezzo-soprano appeared meddle length of vocal folds, while all the soprano appeared short vocal folds. Finally, 9 baritones appeared meddle length of vocal folds, while 2 baritones appeared long vocal folds. In terms of the shape of the vocal folds in female singers, 1 contralto, 5 mezzo-soprano and 4 soprano appeared rounded shape of vocal folds. 6 contralto, 7 mezzo-soprano and 12 soprano appeared elongated vocal folds (Table 8).

Concerning the width of the vocal folds of the male singers, all of the contralto appeared wide vocal fold, all of the mezzo-soprano appeared medium size vocal folds and 8 all of the soprano appeared narrow vocal folds. The thickness of the vocal folds was found to be thin in all of the soprano. All of the mezzo-soprano appeared average thickness of the vocal folds and all of the contralto appeared thick vocal folds (Table 9).

Table 5. Statistical analysis results: correlation between male singers and the variables; tone of speech and color of speech.

\begin{tabular}{cccccccc}
\hline voice category & \multicolumn{4}{c}{ tone of speech } & \multicolumn{3}{c}{ color of speech } \\
\hline & heavy & average & acute & total & Deep & high-toned & total \\
\hline basso & 16 & 0 & 0 & 16 & 3 & 4 & 7 \\
& $100 \%$ & $0 \%$ & $0 \%$ & $100 \%$ & $42.90 \%$ & $57.10 \%$ & $100 \%$ \\
batitones & 2 & 9 & 0 & 11 & 4 & 8 & 12 \\
& $18.20 \%$ & $81.20 \%$ & $0 \%$ & $100 \%$ & $33.30 \%$ & $66.70 \%$ & $100 \%$ \\
tenors & 0 & 0 & 8 & 8 & 6 & 10 & 16 \\
& $0 \%$ & $0 \%$ & $100 \%$ & $100 \%$ & $37.50 \%$ & $62.50 \%$ & $100 \%$ \\
Pearson Chi-square & & 60.45 & & & & 0.173 & \\
p-level & & 0 & & & & 0.917 & \\
\hline
\end{tabular}


Table 6. Statistical analysis results: correlation between female singers and the variables; reliability of the nasal cavity, size of stomatopharynx.

\begin{tabular}{cccccccc}
\hline voice category & \multicolumn{3}{c}{ reliability of nasal cavity } & \multicolumn{3}{c}{ size of stomatopharynx } \\
\hline & good & reduced & total & small & mean & large & total \\
\hline contralto & 2 & 5 & 7 & 0 & 0 & 7 & 7 \\
mezzosoprano & $28.60 \%$ & $71.40 \%$ & $100 \%$ & $0 \%$ & $0 \%$ & $100 \%$ & $100 \%$ \\
soprano & $43.30 \%$ & $66.70 \%$ & $100 \%$ & $0 \%$ & $100 \%$ & $0 \%$ & $100 \%$ \\
Pearson Chi-square & 13 & 3 & 16 & 16 & 0 & 0 & 12 \\
p-level & $81 \%$ & $19 \%$ & $100 \%$ & $100 \%$ & $0 \%$ & $0 \%$ & $100 \%$ \\
\hline
\end{tabular}

Table 7. Statistical analysis results: correlation between female singers and the variables; shape of hard palate, shape of epiglottis.

\begin{tabular}{cccccccc}
\hline voice category & \multicolumn{3}{c}{ shape of hard palate } & \multicolumn{3}{c}{ shape of epiglottis } \\
\hline & oval & arched & flat & total & high & lower & total \\
\hline contralto & 5 & 0 & 2 & 7 & 1 & 6 & 7 \\
mezzosoprano & $31 \%$ & $0 \%$ & $29 \%$ & $100 \%$ & $14 \%$ & $86 \%$ & $100 \%$ \\
soprano & $25 \%$ & $58 \%$ & $17 \%$ & $100 \%$ & $67 \%$ & $33 \%$ & $100 \%$ \\
Pearson Chi-square & 2 & 5 & 9 & 16 & 10 & 6 & 16 \\
p-level & $13 \%$ & $31 \%$ & $56 \%$ & $100 \%$ & $63 \%$ & $37 \%$ & $100 \%$ \\
& & 13.53 & & & & 5.68 & \\
\hline
\end{tabular}

Table 8. Statistical analysis results: correlation between female singers and the variables; length of vocal folds, shape of vocal folds.

\begin{tabular}{cccccccc}
\hline voice category & \multicolumn{3}{c}{ length of vocal folds } & \multicolumn{3}{c}{ shape of vocal folds } \\
\hline & short & meedle & long & total & rounded & enlogated & total \\
\hline contralto & 0 & 0 & 7 & 7 & 1 & 6 & 7 \\
mezzosoprano & $0 \%$ & $0 \%$ & $100 \%$ & $100 \%$ & $14.30 \%$ & $85.70 \%$ & $100 \%$ \\
soprano & $0 \%$ & $100 \%$ & $0 \%$ & $100 \%$ & $41.70 \%$ & $58.30 \%$ & $100 \%$ \\
Pearson Chi-square & 16 & 0 & 0 & 16 & 4 & 12 & 16 \\
p-level & $100 \%$ & $0 \%$ & $0 \%$ & $100 \%$ & $25.00 \%$ & $75.00 \%$ & $100 \%$ \\
\hline
\end{tabular}

The tone of speech was heavy in all of the contralto. Average tone of speech appeared all of the mezzo-soprano, while all of the soprano appeared acute tone 
of speech. Finally, in observing the color of each participant's speech, it was found that 3 contralto, 4 mezzo-soprano and 6 soprano appeared deep voice color. 4 contralto, 8 mezzo-soprano and 10 soprano appeared high-tone voice color (Table 10).

\section{Discussion}

This study attempted to correlate the vocal category of professional singers with vocal folds characteristics, morphometrically, using video laryngeal stroboscopy, on professional singers. This was achieved through the collection between these characteristics and the voice categories of 70 healthy singers. The male singers included basses, baritones and tenors and the female singers were contraltos, mezzo-sopranos and soprano. We observe a relation between the size of stomatopharynx and the voice category. In particular, we observe that as the frequency of the voice in males singers increases, the size of the oropharynx decreases. This relation is inversely proportional, in female singers.

Table 9. Statistical analysis results: correlation between female singers and the variables; width of vocal folds, thickness of vocal folds.

\begin{tabular}{cccccccccc}
\hline voice category & \multicolumn{3}{c}{ width of vocal folds } & \multicolumn{3}{c}{ thikness of vocaal folds } \\
\hline & wide & meedle & narrow & total & thin & average & thick & total \\
\hline contralto & 0 & 7 & 0 & 7 & 0 & 7 & 0 & 7 \\
& $0.00 \%$ & $100 \%$ & $0.00 \%$ & $100 \%$ & $0.00 \%$ & $100 \%$ & $0.00 \%$ & $100 \%$ \\
mezzosoprano & 0 & 0 & 12 & 12 & 0 & 0 & 12 & 12 \\
soprano & $0.00 \%$ & $0.00 \%$ & $100 \%$ & $100 \%$ & $0 \%$ & $0.00 \%$ & $100.00 \%$ & $100 \%$ \\
& 16 & 0 & 0 & 16 & 16 & 0 & 0 & 16 \\
Pearson Chi-square & $100 \%$ & $0 \%$ & $0 \%$ & $100 \%$ & $100 \%$ & $0 \%$ & $0 \%$ & $100 \%$ \\
p-level & & 54.69 & & & & 70 & & \\
\hline
\end{tabular}

Table 10. Statistical analysis results: correlation between female singers and the variables; tone of speech and color of speech.

\begin{tabular}{cccccccc}
\hline voice category & \multicolumn{3}{c}{ tone of speech } & \multicolumn{3}{c}{ colour of speech } \\
\hline & heavy & average & acute & total & deep & high-toned & total \\
\hline contralto & 7 & 0 & 0 & 7 & 3 & 4 & 7 \\
mezzosoprano & $100 \%$ & $0 \%$ & $0 \%$ & $100 \%$ & $42.90 \%$ & $57.10 \%$ & $100 \%$ \\
& 0 & 12 & 0 & 11 & 4 & 8 & 12 \\
soprano & $0.00 \%$ & $100.00 \%$ & $0 \%$ & $100 \%$ & $33.30 \%$ & $66.70 \%$ & $100 \%$ \\
Pearson Chi-square & 0 & 0 & 16 & 8 & 6 & 10 & 16 \\
p-level & $0 \%$ & $0 \%$ & $100 \%$ & $100 \%$ & $37.50 \%$ & $62.50 \%$ & $100 \%$ \\
\hline
\end{tabular}


We also observe that the basso singers mostly appeared oval-shapes hard palate, while most tenors appeared flat hard palate. So, the low frequency male vocal categories relate with oval-shape hard palate, while the high frequency male voices relate with flat shape of hard palate.

We concluded that as the frequency of the male voice increases, the width of the vocal cords decreases.

In addition, as the frequency of the voice increases, the thickness of the vocal cords also increases, both in male and female singers. Therefore, we concluded that as the frequency of the singing voice increases, the tone of voice change in a proportional way.

There is an obvious correlation between the length of the vocal cords and the frequency of the voice in male and female singers. In particular, the frequency of the voice increases as the length of the vocal cords increases. Our results provide support to other studies reporting that statistically significant correlations occur between voice category and the length of the vocal folds.

Moreover, in this study, a statistically significant difference was observed between male singers and the body height. Specifically, basso singers appeared the highest average body height, followed by the tenors and finally the baritones, reinforcing the results of other researchers. Furthermore, we correlated the average body mass with voice category. Statistically significant differences were observed between the weight $[\mathrm{F}=28.76, \mathrm{p}=0.000]$ and the difference was between bass and baritone $[p=0.001]$, bass and tenor $[p=0.000]$ and between baritone and tenor $[\mathrm{p}=0.009]$. Our result comes to an agreement with other studies reporting similar conclusions [17] [50] [51].

Sample size may was a limitation in this study. Due to the pandemic COVID-19 restriction laws, it was not available to recruit more participants. Video laryngeal stroboscopy, considered being a subjective evaluation method, for the assessment of larynx. Using a more objective evaluation method, that requires specific measurements of the dimensions of the vocal folds, would probably be more appropriate to deliver clear and concrete results. Nevertheless, the quality characteristics of voice and larynx, such as the tone of speech, the color of speech as well as the shape of epiglottis, remain features that need the subjective evaluation of the examiner. Taking into account these effect method limitations, the results of this study are good but more objective methods would increase their validity.

\section{Conflicts of Interest}

All authors declare no conflicts of interest in this manuscript.

\section{References}

[1] Sundberg, J. (1977) The Acoustics of the Singing Voice. Scientific American, 236, 82-91. https://doi.org/10.1038/scientificamerican0377-82

[2] Mendes, A.P., Rothman, H.B., Sapienza, C. and Brown, W. (2003) Effects of Vocal Training on the Acoustic Parameters of the Singing Voice. Journal of Voice, 17, 529-543. https://doi.org/10.1067/S0892-1997(03)00083-3 
[3] Adessa, M., Stadelman-Cohen, T., Zipse, L., Guarino, A.J. and Heaton, J.T. (2018) Factors Affecting Voice Therapy Completion in Singers. Journal of Voice, 32, 564571. https://doi.org/10.1016/j.jvoice.2017.06.021

[4] Gray, C.C. (2018) Relationship between Vocal Fatigue and Physical/Psychological Factors in Prospective Vocal Professionals. Master Thesis.

[5] Bullack, A., Gass, C., Nater, U.M. and Kreutz, G. (2018) Psychobiological Effects of Choral Singing on Affective State, Social Connectedness, and Stress: Influences of Singing Activity and Time Course. Frontiers in Behavioral Neuroscience, 12, 22. https://doi.org/10.3389/fnbeh.2018.00223

[6] Ray, C., Trudeau, M.D. and McCoy, S. (2018) Effects of Respiratory Muscle Strength Training in Classically Trained Singers. Journal of Voice, 32, 644e25-644e34. https://doi.org/10.1016/j.jvoice.2017.08.005

[7] Zimmermann, D. (1938) Die Mazung der Stimmlippenlange bei Sangern und Sangerinnen. Arch Sprach u Stimmheilk, 2, 103.

[8] Nguyen, A. (2015) How Vocal Classification Affects Young Singers. https://doi.org/10.15760/honors.129

[9] Miller, R. (2000) Training Soprano Voices. Oxford University Press, USA.

[10] Johnson, A.M. and Kempster, G.B. (2011) Classification of the Classical Male Singing Voice Using Long-Term Average Spectrum. Journal of Voice, 25, 538-543. https://doi.org/10.1016/j.jvoice.2010.05.009

[11] Sonninen, A. and Hurme, P. (1998) Vocal Fold Strain and Vocal Pitch in Singing Radiographic Observations of Singers and Non-Singers. Journal of Voice, 12, 274-286. https://doi.org/10.1016/S0892-1997(98)80018-0

[12] Yamasaki, R., Behlau, M., do Brasil, O.D.O.C. and Yamashita, H. (2011) MRI Anatomical and Morphological Differences in the Vocal Tract between Dysphonic and Normal Adult Women. Journal of Voice, 25, 743-750. https://doi.org/10.1016/j.jvoice.2010.08.005

[13] Wilson, S.J., Abbott, D.F., Lusher, D., Gentle, E.C. and Jackson, G.D. (2011) Finding Your Voice: A Singing Lesson from Functional Imaging. Human Brain Mapping, 32, 2115-2130. https://doi.org/10.1002/hbm.21173

[14] Vorik, A., Unteregger, F., Zwicky, S., Schiwowa, J., Potthast, S. and Storck, C. (2017) Three-Dimensional Imaging of High-Resolution Computer Tomography of Singers' Larynges-A Pilot Study. Journal of Voice, 31, 115e17-115e21. https://doi.org/10.1016/j.jvoice.2016.03.011

[15] Vos, R.R., Murphy, D.T., Howard, D.M. and Daffern, H. (2018) Determining the Relevant Criteria for Three-Dimensional Vocal Tract Characterization. Journal of Voice, 32, 130-142. https://doi.org/10.1016/j.jvoice.2017.04.001

[16] Cho, W., Hong, J. and Park, H. (2012) Real-Time Ultrasonographic Assessment of True Vocal Fold Length in Professional Singers. Journal of Voice, 26, 819e1-6. https://doi.org/10.1016/j.jvoice.2012.05.007

[17] Mürbe, D., Roers, F. and Sundberg, J. (2011) Voice Classification in Professional Singers: The Influence of Vocal Fold Length, Vocal Tract Length and Body Measurements. $H N O$, 59, 556-562. https://doi.org/10.1007/s00106-011-2304-1

[18] Clarós, P., Sobolewska, A.Z., Doménech-Clarós, A., Clarós-Pujol, A., Pujol, C. and Clarós, A. (2019) CT-Based Morphometric Analysis of Professional Opera Singers' Vocal Folds. Journal of Voice, 33, 583.e1-583.e8. https://doi.org/10.1016/j.jvoice.2018.02.010

[19] Borgard, H.L., Baab, K., Pasch, B. and Riede, T. (2019) The Shape of Sound: A Geo- 
metric Morphometrics Approach to Laryngeal Functional Morphology. Journal of Mammalian Evolution, 27, 577-590. https://doi.org/10.1007/s10914-019-09466-9

[20] Ramli, M.I., Hamzaid, N.A. and Engkasan, J.P. (2019) Monitoring Breathing Muscle Performance during Singing Noninvasively Using Mechanomyography and Electromyography. Journal of Voice, 34, 862-869.

https://doi.org/10.1016/j.jvoice.2019.06.006

[21] Ternström, S., D'Amario, S. and Selamtzis, A. (2018) Effects of the Lung Volume on the Electroglottographic Waveform in Trained Female Singers. Journal of Voice, 34, 485.e1-485.e21.

[22] Debruyne, F., Ostyn, F., Delaere, P. and Wellens, W. (1997) Acoustic Analysis of the Speaking Voice after Thyroidectomy. Journal of Voice, 11, 479-482. https://doi.org/10.1016/S0892-1997(97)80046-X

[23] Štajner-Katušić, S., Horga, D. and Zrinski, K.V. (2008) A Longitudinal Study of Voice before and after Phonosurgery for Removal of a Polyp. Clinical Linguistics \& Phonetics, 22, 857-863. https://doi.org/10.1080/02699200802130813

[24] Al-Yahya, S.N., Muhammad, R., Suhaimi, S.N., Azman, M., Mohamed, A.S. and Baki, M.M. (2019) Selective Laryngeal Examination: Sensitivity of Endocrine Surgeons in Screening Voice Abnormality. Journal of Voice, 34, 811.e13-811.e20.

[25] Petrovic-Lazic, M., Jovanovic, N., Kulic, M., Babac, S. and Jurisic, V. (2015) Acoustic and Perceptual Characteristics of the Voice in Patients with Vocal Polyps after Surgery and Voice Therapy. Journal of Voice, 29, 241-246. https://doi.org/10.1016/j.jvoice.2014.07.009

[26] Chernobelsky, S.I. (2007) The Treatment and Results of Voice Therapy amongst Professional Classical Singers with Vocal Fold Nodules. Logopedics Phoniatrics Vocology, 32, 178-184. https://doi.org/10.1080/14015430600852043

[27] Dejonckere, P.H., Bradley, P., Clemente, P., et al. (2001) A Basic Protocol for Functional Assessment of Voice Pathology, Especially for Investigating the Efficacy of (Phonosurgical) Treatments and Evaluating New Assessment Techniques. European Archives of Oto-Rhino-Laryngology, 258, 77-82. https://doi.org/10.1007/s004050000299

[28] Shih, L.C., Piel, J., Warren, A., Kraics, L., et al. (2012) Singing in Groups for Parkinson's Disease (SING-PD): A Pilot Study of Group Singing Therapy for PD-Related Voice/Speech Disorders. Parkinsonism \& Related Disorders, 18, 548-552. https://doi.org/10.1016/j.parkreldis.2012.02.009

[29] Johns, M.M. (2003) Update on the Etiology, Diagnosis, and Treatment of Vocal Fold Nodules, Polyps, and Cysts. Current Opinion in Otolaryngology \& Head and Neck Surgery, 11, 456-461. https://doi.org/10.1097/00020840-200312000-00009

[30] Hicks, M., Brugman, S.M. and Katial, R. (2008) Vocal Cord Dysfunction/Paradoxical Vocal Fold Motion. Primary Care, 35, 81-103.

https://doi.org/10.1016/j.pop.2007.09.005

[31] Ebersole, B., Soni, R.S., Moran, K., Lango, M., Devarajan, K. and Jamal, N. (2018) The Influence of Occupation on Self-Perceived Vocal Problems in Patients with Voice Complaints. Journal of Voice, 32, 673-680. https://doi.org/10.1016/j.jvoice.2017.08.028

[32] Tafiadis, D., Helidoni, M.E., Chronopoulos, S.K., Kosma, E.I., Ziavra, N. and Velegrakis, G.A. (2020) Cross-Cultural Adaptation and Validation of the Greek Voice Handicap Index-10 (GVHI-10) with Additional Receiver Operating Characteristic Analysis. Journal of Voice, 34, 304.e1-304.e8. https://doi.org/10.1016/j.jvoice.2018.09.009 
[33] Tafiadis, D., Chronopoulos, S.K., Helidoni, M.E., Kosma, E.I., et al. (2019) Checking for Voice Disorders without Clinical Intervention: The Greek and Global VHI Thresholds for Voice Disordered Patients. Scientific Reports, 9, Article No. 9366. https://doi.org/10.1038/s41598-019-45758-Z

[34] Tafiadis, D., Chronopoulos, S.K., Siafaka, V., et al. (2017) Comparison of Voice Handicap Index Scores between Female Students of Speech Therapy and Other Health Professions. Journal of Voice, 31, 583-588.

https://doi.org/10.1016/j.jvoice.2017.01.013

[35] Tafiadis, D., Kosma, E.I., Chronopoulos, S.K., Voniati, L. and Ziavra, N. (2018) A Preliminary Receiver Operating Characteristic Analysis on Voice Handicap Index Results of the Greek Voice-Disordered Patients. International Journal of Otolaryngology and Head \& Neck Surgery, 7, 98-114. https://doi.org/10.4236/ijohns.2018.73013

[36] Sorensen, D. and Horii, Y. (1982) Cigarette Smoking and Voice Fundamental Frequency. Journal of Communication Disorders, 15, 135-144. https://doi.org/10.1016/0021-9924(82)90027-2

[37] Tafiadis, D., Toki, E.I., Miller, K.J. and Ziavra, N. (2017) Effects of Early Smoking Habits on Young Adult Female Voices in Greece. Journal of Voice, 31, 728-732. https://doi.org/10.1016/j.jvoice.2017.03.012

[38] Kusama, T. and Ota, K. (2002) Radiological Protection for Diagnostic Examination of Pregnant Women. Congenital Anomalies, 42, 10-14. https://doi.org/10.1111/j.1741-4520.2002.tb00848.x

[39] Schwarz, G.S. (1968) Radiation Hazards to the Human Fetus in Present-Day Society. Should a Pregnant Woman Be Subjected to a Diagnostic X-Ray Procedure? Bulletin of the New York Academy of Medicine, 44, 388-399.

[40] Simo, R., Bradley, P., Chevalier, D., Dikkers, F., Eckel, H., Matar, N., Quer, M., et al. (2014) European Laryngological Society: ELS Recommendations for the Follow-Up of Patients Treated for Laryngeal Cancer. European Archives of Oto-Rhino-Laryngology, 271, 2469-2479. https://doi.org/10.1007/s00405-014-2966-X

[41] Nospes, S., Kuhr, K., Napiontek, U. and Keilmann, A. (2011) Stroboskopie-Befunde: Flexible CCD-Videostroboskopie und klassische Lupenstroboskopie im Vergleich. Laryngo-Rhino-Otologie, 90, 218-223. https://doi.org/10.1055/s-0031-1271631

[42] Sachdeva, K., Mittal, N. and Sachdeva, N. (2020) Role of Video Laryngostroboscopy in Benign Disease of Larynx. Indian Journal of Otolaryngology and Head \& Neck Surgery, 72, 267-273. https://doi.org/10.1007/s12070-020-01827-8

[43] Mehta, D.D. and Hillman, R.E. (2012) Current Role of Stroboscopy in Laryngeal Imaging. Current Opinion in Otolaryngology \& Head and Neck Surgery, 20, 429436. https://doi.org/10.1097/MOO.0b013e3283585f04

[44] Colton, R.H., Woo, P., Brewer, D.W., Griffin, B. and Casper, J. (1995) Stroboscopic Signs Associated with Benign Lesions of the Vocal Folds. Journal of Voice, 9, 312-325. https://doi.org/10.1016/S0892-1997(05)80240-1

[45] Kelley, R.T., Colton, R.H., Casper, J., Paseman, A. and Brewer, D. (2011) Evaluation of Stroboscopic Signs. Journal of Voice, 25, 490-495. https://doi.org/10.1016/j.jvoice.2010.03.004

[46] Harris, L.D. (1996) A Study of Intensity Control in Males with Developing Voices: Implications for Pitch Range and Tessitura. University of North Texas ProQuest Dissertations Publishing, Denton.

[47] Haynes, B. (2002) A History of Performing Pitch: The Story of "A". Scarecrow Press, Lanham. 
[48] Barcan, L.J. (2013) Tessitura Changes in Music Theatre Repertoire for the Soprano Voice. Proceedings of 8 th International Congress of Voice Teachers, Brisbane, 10-14 July 2013, 28-42.

[49] Murrell, G.L. (2013) Components of the Nasal Examination. Aesthetic Surgery Journal, 33, 38-42. https://doi.org/10.1177/1090820X12469626

[50] Roers, F. (2009) Voice Classification and Vocal Tract of Singers: A Study of X-Ray Images and Morphology. The Journal of the Acoustical Society of America, 125, 503-512. https://doi.org/10.1121/1.3026326

[51] Roers, F., Mürbe, D. and Sundberg, J. (2009) Predicted Singers' Vocal Fold Lengths and Voice Classification-A Study of X-Ray Morphological Measures. Journal of Voice, 23, 408-413. https://doi.org/10.1016/j.jvoice.2007.12.003 